\title{
IASI temperature and water vapor retrievals - error assessment and validation
}

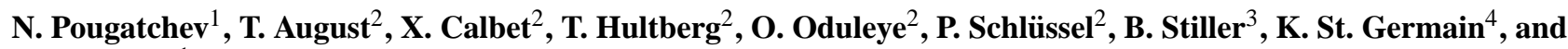 \\ G. Bingham ${ }^{1}$ \\ ${ }^{1}$ Space Dynamics Laboratory, North Logan, Utah, USA \\ ${ }^{2}$ EUMETSAT, Darmstadt, Germany \\ ${ }^{3}$ German Meteorological Service, Meteorological Observatory, Lindenberg, Germany \\ ${ }^{4}$ Integrated Project Office, Silver Spring, Maryland, USA
}

Received: 24 February 200 - Published in Atmos. Chem. Phys. Discuss.: 25 March 2009

Revised: 28 July 2009 - Accepted: 16 August 2009 - Published: 8 September 2009

\begin{abstract}
The METOP-A satellite Infrared Atmospheric Sounding Interferometer (IASI) Level 2 products comprise retrievals of vertical profiles of temperature and water vapor. The error covariance matrices and biases of the most recent version (4.3.1) of the L2 data were assessed, and the assessment was validated using radiosonde data for reference. The radiosonde data set includes dedicated and synoptic time launches at the Lindenberg station in Germany. For optimal validation, the linear statistical Validation Assessment Model (VAM) was used. The VAM uses radiosonde profiles as input and provides optimal estimate of the nominal IASI retrieval by utilizing IASI averaging kernels and statistical characteristics of the ensembles of the reference radiosondes. For temperatures above $900 \mathrm{mb}$ and water retrievals above $700 \mathrm{mb}$, level expected and assessed errors are in good agreement. Below those levels, noticeable excess in assessed error is observed, possibly due to inaccurate surface parameters and undetected clouds/haze.
\end{abstract}

\section{Introduction}

Atmospheric sounders, i.e., systems that remotely measure atmospheric thermodynamic parameters and constituents, are important sources of data for numerous practical and scientific applications such as Numeric Weather Prediction (NWP) and climate studies. To be usable, the data from

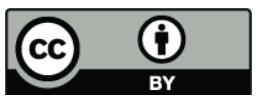

Correspondence to: N. Pougatchev (nikita.pougatchev@sdl.usu.edu) satellite sounders must be validated in the sense that their relation to the true state of the atmosphere must be known with statistically estimated error (Rodgers, 2000 and Eyre, 1997). Thus, we define validation as an activity that estimates the error of the sounder during its operation.

In the context of current work, the term atmospheric sounder implies a satellite-borne measurement system comprising a sensor and subsequent data processing. The sensor receives and transforms upwelling radiance, and the data processing generates calibrated spectra and retrievals of atmospheric parameters. In the process of designing, prelaunch testing, and calibrating a measurement system, modeled (nominal) relations between the true state and measurement results are established. Following Clive Rodgers (2000), we call this characterization and error analysis. After launch, the actual errors of measurements in the real atmosphere may differ from the errors established during prelaunch analysis. That difference may be caused by various factors such as changes in the instrument performance or inaccuracy in atmospheric radiative transfer modeling. Thus, special efforts should be made to validate the pre-launch error assessment during the in-orbit phase of the satellite system. It is pertinent to note that both measured radiances and retrieved atmospheric state are used for practical applications; hence, errors of both products have to be validated. In the present work we address the retrievals using the approach which is also applicable to the validation of radiances (Pougatchev, 2008).

One way to validate the satellite data is to perform a proper comparison of the satellite data with their estimate based on independently acquired reference data set. The reference

Published by Copernicus Publications on behalf of the European Geosciences Union. 
(validating) system can be air-borne in situ, e.g. radiosondes (Tobin et al., 2006; Miglioniri et al., 2004), air-borne remote (Smith, 2005), or space-borne (Lary and Lait, 2006; GSICS, 2007). Because a remote sounder measures some function of the atmosphere-surface state (Rodgers, 2000), the ideal validation would be a straightforward comparison of the data from the system to be validated with the data from a validating system that samples exactly the same atmospheric state and has identical characterization but negligible errors. Unfortunately, on many occasions this approach is not feasible. As a rule, the systems have different characteristics, nonnegligible errors, and perform their measurements at close but different times and locations. We will call these types of measurements correlative measurements.

Rodgers and Connor (2003) demonstrated that even when two different systems perform the measurements on the same state of the atmosphere, a sensible comparison cannot be reduced to a straightforward, point-by-point analysis of differences; proper statistical methods should be used instead to reconcile what we will call characteristic difference error. They developed an approach that has been applied to validation of the MIPAS ozone and MOPITT carbon monoxide satellite measurements (Barret et al, 2003).

In practice, the situation is more complex than considered in previous research (Rodgers, 2000; Rodgers and Connor, 2003), i.e., the compared systems perform their measurements at different times and locations. In spatially nonuniform and dynamic atmosphere, that fact causes what we will call state non-coincidence error. For the AIRS validation, Tobin et al. (2006) resolved this issue by using a multi-instrument/platform correlative measurement data set and building the best estimate of the true atmospheric state for each individual AIRS measurement used for the validation. The method is sufficiently accurate, but, unfortunately, all the instruments must be present at the same site (in this case it is ARM site in Oklahoma, USA), which significantly limits its applicability.

For the most accurate and comprehensive assessment of the IASI performance during the early stage of its operation Joint Airborne IASI Validation Experiment (JAIVEx) has been performed in April-May of 2007. Some results related to the retrieval products are presented in (Larar et al., 2009; Liu et al., 2009; Zhou et al., 2009).

In the current work, we use the approach developed by Pougatchev (2008), which does not require other measurements besides the correlative data per se. The best estimate of the true atmospheric state and corresponding nominal satellite measurement are provided by the linear statistical Validation Assessment Model (VAM). For this particular study, the VAM uses correlative radiosonde profiles as input and returns the optimal estimate of the nominal IASI retrieval by utilizing IASI averaging kernels and statistical characteristics of the ensembles of the reference radiosondes.

In the following sections, we will briefly describe the VAM and its application to the specific data sets (Sect. 2); and the results of the assessment of the IASI temperature and water vapor retrieval errors in the form that can be utilized by the community - regionally specific covariance and bias (Sect. 3). We will mostly follow the terminology and notations used by Rodgers (1976, 1990, 2000). In particular, bold lower case symbols denote column vectors; upper case bold typeface denotes matrices, and regular italicized typeface is reserved for scalars.

\section{Error assessment and validation technique}

In this section we will present the key formulas used by the VAM as well as techniques we used to estimate temporal and spatial statistical characteristics of the reference ensemble of the atmospheric states. A detailed discussion of the approach and a description of the VAM can be found in Pougatchev (2008).

\subsection{Basic relations}

We assume that the validated IASI sounder performs its measurements on the ensemble of the true state of $\mathbf{X}_{v}$, which has mean value $\overline{\mathbf{x}}_{\mathbf{v}}$ and covariance $\mathbf{S}_{\mathbf{v}}$. The retrieved profile $\hat{\mathbf{x}}$ in linear approximation is related to the true state $\mathbf{x}_{\mathbf{v}} \in \mathbf{X}_{\mathbf{v}}$ as follows:

$\hat{\mathbf{x}}=\mathbf{x}_{\mathbf{a}}+\mathbf{A}\left(\mathbf{x}_{\mathbf{v}}-\mathbf{x}_{\mathbf{a}}\right)+\varepsilon$

where $\mathbf{x}_{\mathbf{a}}$ is the a priori profile (linearization point); $\mathbf{A}$ is the averaging kernel matrix (Freché derivatives); and $\varepsilon$ is the error that we will assess through validation. The error may be caused by various factors; in particular, there is contribution from inevitable noise in the radiances measured by the sounder. This component is called retrieval noise, and it gives the lower estimate of the total retrieval error. Alternatively, the error of retrieval can be defined as the difference between the true and retrieved profiles, i.e., total retrieval error:

$\varepsilon_{\text {tot }}=\hat{\mathbf{x}}-\mathbf{x}_{\mathbf{v}}=(\mathbf{I}-\mathbf{A})\left(\mathbf{x}_{\mathbf{a}}-\mathbf{x}_{\mathbf{v}}\right)+\varepsilon$

We present the total retrieval error in the following form:

$\begin{array}{ll}\varepsilon_{\text {tot }}=(\mathbf{I}-\mathbf{A})\left(\mathbf{x}_{\mathbf{a}}-\mathbf{x}_{\mathbf{v}}\right) & \text { smoothing error } \varepsilon_{\mathbf{s m}} \\ +\varepsilon_{\mathbf{n}} & \text { retrieval noise } \\ +\varepsilon_{\mathbf{u n}} & \text { unmodeled error }\end{array}$

From pre-flight algorithm characterization, we know the expected averaging kernels $\mathbf{A}$; hence, given the covariance of the ensemble of true states $\mathbf{S}_{\mathbf{v}}$, the covariance of the expected error $\mathbf{S}_{\text {tot_e }}$ is:

$\mathbf{S}_{\text {tot_e }}=(\mathbf{I}-A) \mathbf{S}_{\mathbf{v}}(\mathbf{I}-\mathbf{A})^{T}+\mathbf{S}_{\mathbf{n}}=\mathbf{S}_{\mathbf{s m}}+\mathbf{S}_{\mathbf{n}}$ 
In Sect. 2.2 we discuss the technique for estimating $\mathbf{S}_{\mathbf{n}}$ from satellite data only. Later in this section we provide the basic formulas for assessing the possible unmodeled error through comparing the retrievals to correlative sondes.

In the context of this paper, the term $\mathbf{x}_{\mathbf{a}}+\mathbf{A}\left(\mathbf{x}_{\mathbf{v}}-\mathbf{x}_{\mathbf{a}}\right)$ in Eq. (1) represents the expected retrieval. We will estimate it from correlative radiosonde measurements using the VAM.

We assume that the radiosonde performs correlative measurements on the ensemble of true states $\mathbf{X}_{\mathbf{c}}$, and returns profile $\mathbf{x}_{\mathbf{S}}$, which is related to the true state $\mathbf{x}_{\mathbf{c}} \in \mathbf{X}_{\mathbf{c}}$ as follows:

$\mathbf{x}_{\mathbf{s}}=x_{\mathbf{c}}+\varepsilon_{\mathbf{c}}$

The correlative ensemble has mean value $\overline{\mathbf{x}}_{\mathbf{c}}$, covariance $\mathbf{S}_{\mathbf{c}}$, and noise covariance $\mathbf{S}_{\varepsilon c}$.

Following the formalism from Pougatchev (2008), we can write

$\left(\mathbf{x}_{\mathbf{v}}-\overline{\mathbf{x}}_{\mathbf{v}}\right)=\mathbf{B}_{\mathbf{x}}\left(\mathbf{x}_{\mathbf{c}}-\overline{\mathbf{x}}_{\mathbf{c}}\right)+\xi$

where correlation matrix $\mathbf{B}_{\mathbf{x}}$ and random error $\xi$ depend on temporal and spatial non-coincidence between satellite and sonde measurements. The error $\xi$ has zero mean value and covariance $\mathbf{S}_{\xi}$. The term $\mathbf{B}_{\mathbf{x}}\left(\mathbf{x}_{\mathbf{c}}-\overline{\mathbf{x}}_{\mathbf{c}}\right)$ is the best linear estimate of the variation of the satellite true state; the error of the estimate is $\xi$. In this context, the best estimate is used in the sense of expected value.

For practical validation we consider the difference:

$\delta=\hat{\mathbf{x}}-\mathbf{A B} \mathbf{x}_{\mathbf{s}}$

It can be demonstrated that covariance of the difference in the absence of residual/unmodelled error is

$\mathbf{S}_{\delta}=\mathbf{A} \mathbf{S}_{\xi} \mathbf{A}^{T}+\mathbf{S}_{\mathbf{n}}+(\mathbf{A B}) \mathbf{S}_{\varepsilon \mathbf{c}}(\mathbf{A B})^{T}$

The term $\mathbf{A} \mathbf{S}_{\xi} \mathbf{A}^{T}$ accounts for non-coincidence error, $\mathbf{S}_{\mathbf{n}}$ represents retrieval noise, and $(\mathbf{A B}) \mathbf{S}_{\varepsilon \mathbf{c}}(\mathbf{A B})^{T}$ represents the contribution of the radiosonde error in the comparison. Thus, to validate the error assessment, we will calculate the sample covariance $\tilde{\mathbf{S}}_{\delta}$, and then the covariance $\tilde{\mathbf{S}}_{\mathbf{n}}$

$\tilde{\mathbf{S}}_{\mathbf{n}}=\tilde{\mathbf{S}}_{\delta}-\mathbf{A} \mathbf{S}_{\xi} \mathbf{A}^{T}-(\mathbf{A B}) \mathbf{S}_{\varepsilon \mathbf{c}}(\mathbf{A B})^{T}$

accounts for both retrieval noise and possible unmodeled error.

In Eq. (9) the first term on its right side is calculated from correlative measurements. The other terms are inferred from the sounder characterization and radiosonde error (averaging kernels $\mathbf{A}$ and noise covariance $\mathbf{S}_{\varepsilon \mathbf{c}}$ ) and from statistical analysis of the correlative ensemble (correlative matrix $\mathbf{B}$ and non-coincidence error covariance $\mathbf{S}_{\xi}$ ).

Thus, Eq. (4) provides us with the expected error assessment, whereas the value from Eq. (9) validates the expected assessment. In the following sections, we present the practical application of this methodology to the IASI validation campaign at Lindenberg station in June-August 2007.

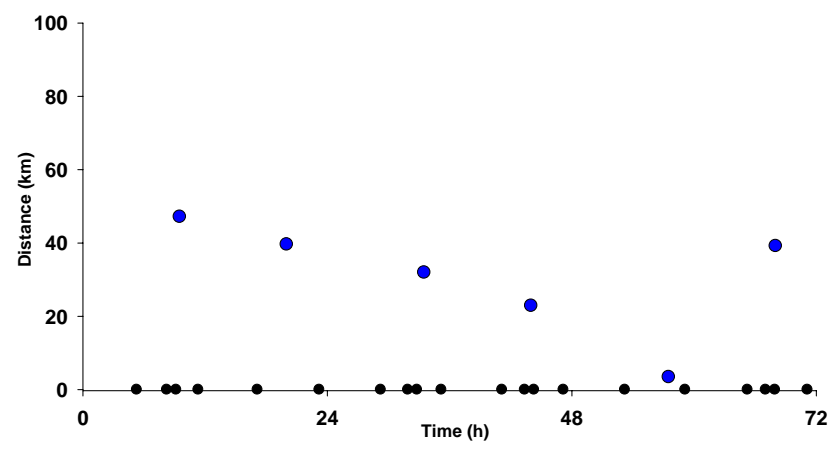

Fig. 1. Spatial-temporal scheme of radiosonde launches - black circles, and satellite overpasses - blue circles denote average distance of individual FOVs within an overpass.

\subsection{Estimation of non-coincidence errors and retrieval noise}

Covariances for spatial and temporal non-coincidence errors as well as the correlation matrix $\mathbf{B}$ are needed for accurate validation (see Eqs. 7-9). Temporal non-coincidence errors and associated correlative matrices are derived from statistical analysis of the radiosonde profiles (Pougatchev, 2008). Spatial non-coincidence error and retrieval noise are inferred from actual IASI retrievals.

Radiosonde launches for the validation campaign and IASI individual FOV retrievals are schematically presented in Fig. 1 (for clarity we show only three days out of a total of 92). Time is counted from the beginning of the validation campaign (1 June 2007). Distance $d$ is the distance of an overpass' retrievals from radiosonde site. Blue circles indicate the average distance. Because we consider only cloud free FOV retrievals, the sample size as well as distance vary from overpass to overpass. Radiosonde profiles are organized in a time series $\left\{\mathbf{x}\left(t_{i}\right)\right\}$. For a given non-coincidence $\tau$ sample auto-covariance matrices $\mathbf{S}(\tau)$ and $\mathbf{S}(0)$ are calculated. Then matrices B and $\mathbf{S}_{\xi}$ are derived using the following relations:

$$
\begin{aligned}
& \mathbf{B}(\tau)=S(\tau) \mathbf{S}^{-1}(0) \\
& \mathbf{S}_{\xi}(\tau)=\mathbf{S}(0)-\mathbf{B}(\tau) \mathbf{S}(0) \mathbf{B}^{T}(\tau)
\end{aligned}
$$

Detailed discussion on practical aspects such as organizing the time series, removing the seasonal variation, and covariance inversion, etc. can be found in Pougatchev (2008).

Consider the satellite making a measurement at a moment $t$ and distance $d$ from the radiosonde over the true state:

$\mathbf{x}(t, d)=\mathbf{x}(t, 0)+\xi_{d}$,

where $\xi(d)$ is random spatial non-coincidence error with zero mean and covariance $\mathbf{S}_{\xi}(d)$. Then the Eq. (6) can be rewritten as follows:

$(\overline{\mathbf{x}}-x(t, d))=\mathbf{B}(\tau)(\overline{\mathbf{x}}-x(t+\tau, 0))+\xi_{\tau}+\xi_{d}$. 


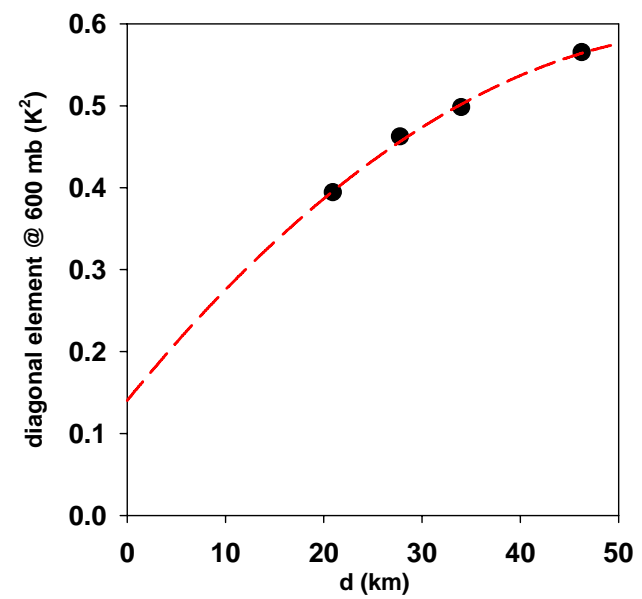

Fig. 2. Red dashed line is quadratic approximation of diagonal elements of the temperature structure function $\mathbf{D}(\mathrm{d})$ - black circles. Intercept provides doubled estimate of the retrieval noise.

Equation (8) takes the form:

$\mathbf{S}_{\delta}=\mathbf{A} \mathbf{S}_{\xi}(\tau) \mathbf{A}^{T}+\mathbf{A} \mathbf{S}_{\xi}(d) \mathbf{A}^{T}+\mathbf{S}_{\mathbf{n}}+\left(\mathbf{A B}(\tau) \mathbf{S}_{\varepsilon \mathbf{c}}(\mathbf{A B}(\tau))^{T}\right.$.

To analyze the spatial non-coincidence error, we used the actual IASI retrievals. We considered retrievals within a $100-\mathrm{km}$ radius around the station. The sample of the retrievals was organized in a set $\left\{\mathbf{x}\left(\mathbf{z}_{i j}\right)\right\}$, where $i=1,2, \ldots, N$ indicates the $i$-th overpass selected for validation, and $j=1,2, \ldots, M_{i}$ is the index of individual Field Of View (FOV) retrieval within $i$-th overpass; $\mathbf{z}_{i j}$ is geolocation of the retrieval. For a given $i$-th overpass sample, second-order structure function $\mathbf{D}_{i}(d)$ was calculated. The structure function for the validation ensemble $\mathbf{D}(d)$ was calculated by averaging individualD $\mathbf{D}_{i}(d)$ over time:

$\mathbf{D}(d)=\sum_{i=1}^{N} \mathbf{D}_{i}(d)$

We present $\mathbf{D}(d)$ as the sum of the estimate of the retrieval noise $\hat{\mathbf{S}}_{\mathbf{n}}$ and the non-coincidence error term $\hat{\mathbf{S}}_{\xi}(d)$ :

$\mathbf{D}(d)=2 \hat{\mathbf{S}}_{n}+\hat{\mathbf{S}}_{\xi}(d)$,

with relation $\hat{\mathbf{S}}_{\xi}(0)=\mathbf{0}$. That equation gives us a practical recipe for estimating the retrieval noise. Extrapolation of $\mathbf{D}(d)$ to $d=0$ gives us the estimate of $\mathbf{S}_{\mathbf{n}}$. In this context, retrieval noise accounts for all atmospheric state independent errors in the retrieval. Analogously, $\hat{\mathbf{S}}_{\xi}(d)$ accounts for the spatial non-coincidence error in the sense of Eq. (14) and for any other state-dependent errors of the retrieval. The plot in Fig. 2 illustrates this approach for one of the diagonal elements of the $\mathbf{D}$ matrix for temperature retrievals. For this study we considered dedicated radiosondes launches corresponding to average time non-coincidence $\tau \approx 0.5 \mathrm{~h}$. For the
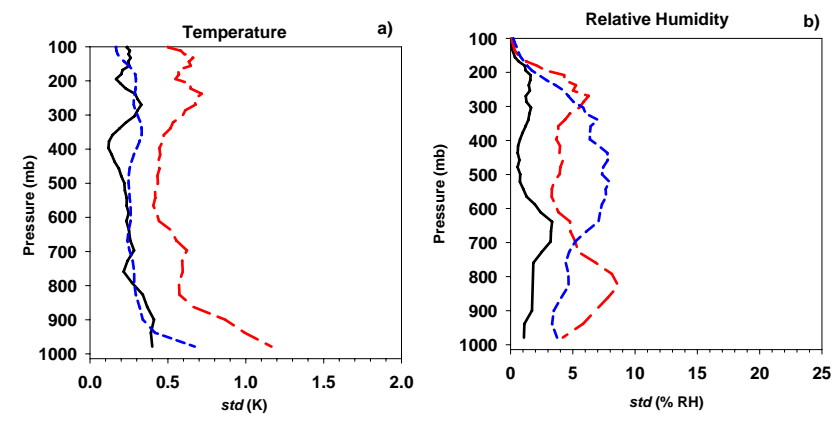

Fig. 3. (a) Temperature retrievals and (b) Relative humidity retrievals. Square roots of diagonal elements of the covariance matrices of the: retrieval noise (solid black lines); temporal non-coincidence of $0.5 \mathrm{~h}$ (dashed blue lines); and spatial noncoincidence error of $20 \mathrm{~km}$ (dashed red lines).

adopted validation scenario average distance of overpass retrievals from the radiosonde launch site is $d \approx 20 \mathrm{~km}$. In Fig. 3 we present the square roots of the diagonal elements of the matrices $\hat{\mathbf{S}}_{\mathbf{n}}, \mathbf{S}_{\xi}(\tau=0.5 \mathrm{~h})$, and $\hat{\mathbf{S}}_{\xi}(d=20 \mathrm{~km})$.

We will use these matrices for validation of the retrievals; therefore, some words are in order to discuss their uncertainties. Partitioning $\mathbf{D}(d)$ into two terms (see Eq. 16) involves extrapolation, which can be done different ways. We investigated the possible uncertainties by using linear and quadratic approximation with different numbers of data points and concluded that the resulting std uncertainty is approximately within $\pm 0.1 \mathrm{~K}$ and $\pm 2 \% \mathrm{RH}$ for $\hat{\mathbf{S}}_{\mathbf{n}}$. Due to relatively small number of sondes selected for validation (33 sondes, selection criteria are in the Section 3.1), covariance of the temporal non-coincidence error $\mathbf{S}_{\xi}(\tau)$ was calculated (Eqs. (10) and (11) involving matrix inversion) for the ensemble comprising all radiosondes during the campaign period ( 650 sondes). The profiles of the whole ensemble exhibit higher variance than the profiles selected for analysis; std approximately $1 \mathrm{~K}$ for temperature and 5\% for RH. These errors may result in overestimation of the temporal non-coincidence error for the particular validation scenario.

\section{Results and discussion}

\subsection{Data description}

The correlative data set covers the time period from 1 July to 31 August 2007. The sondes were launched from Lindenberg station $\left(52.21^{\circ} \mathrm{N}, 14.21^{\circ} \mathrm{E}, 125 \mathrm{~m}\right.$ a.s.l.). The Vaisala RS92-SGP radiosondes were launched at synoptic times 04:45 UTC, 10:45 UTC, 16:45 UTC, and 22:45 UTC, as well as one hour and five minutes prior to IASI overpasses. In the current study, we consider only the dedicated launches. For the sondes total uncertainty in sounding is $0.5 \mathrm{~K}$ for temperature and 5\% for relative humidity (Vaisala 2005). Random std error of the sondes is assumed to be $0.14 \mathrm{~K}$ for any 
temperature below $100 \mathrm{mb}$ and $1.4 \%$ for relative humidity (Vaisala 2009).

The IASI temperature and water vapor profile retrievals are v. 4.3.1 EUMETSAT Level 2 products (Schlüssel et al., 2005; Calbet et al., 2006). The profiles are on standard IASI 90 point pressure grid levels. We applied a flat bias correction that is based on ECMWF model analysis and RTIASI fast radiative transfer (that is used in the L2 processor). There is no connection of the product processing/retrieval to radiosonde measurements. For initial analysis we selected overpasses with cloud-free retrievals (as reported by cloud flags in the product) within $100 \mathrm{~km}$ about the Lindenberg launch site. For each selected overpass (index $i=1,2, \ldots, N$ ) and two altitude ranges (index $j$ ): $(980 \mathrm{mb}-700 \mathrm{mb} ; j=1)$ and ( $700 \mathrm{mb}-50 \mathrm{mb} ; j=2)$ we calculated std of the repeatability of the temperature retrievals averaged over the altitude range $-\sigma_{i j}$. We also calculated the averages over the overpasses $-\sigma_{j}=1 / N \sum_{i=1}^{N} \sigma_{i j} \quad j=1,2$. Then additional filtering of the data was performed, i.e., the $i$-th overpass was rejected if $\sigma_{i j}>3 \sigma_{j}$ where $3 \sigma_{1}=2.5 \mathrm{~K}$ and $3 \sigma_{2}=1.8 \mathrm{~K}$. That rigorous filtering and bad (cloudy and hazy) weather after July 17 yielded 17 overpasses and 33 corresponding sondes selected for final analysis. On average, each selected overpass contains 15 retrieved profiles.

\subsection{Error assessment}

The VAM with the inputs described in Sect. 2.2 was applied to the data described in Sect. 3.1. For temperature and relative humidity retrievals, we estimated bias against radiosondes and total error covariance matrices. The matrices are calculated based on the following relations:

$$
\begin{aligned}
& \mathbf{S}_{\text {tot_exp }}=S_{\mathbf{s} m}+\hat{S}_{\mathbf{n}} \\
& \mathbf{S}_{\text {tot_val }}=S_{\mathbf{s} m}+\tilde{S}_{\mathbf{n}}
\end{aligned}
$$

where $\mathbf{S}_{\mathbf{s m}}$ is smoothing error covariance; $\hat{\mathbf{S}}_{\mathbf{n}}$ is estimated retrieval noise or, in other words, atmospheric state independent error (Eq. 13); and $\tilde{\mathbf{S}}_{\mathbf{n}}$ is covariance matrix which accounts for both retrieval noise and unmodeled state dependent error (Eq. 9). Detailed discussion on the nature of $\tilde{\mathbf{S}}_{\mathbf{n}}$ and its relationship to smoothing errors can be found in Pougatchev (2008).

For temperature, the results are presented in Fig. 3. The assessed and expected total retrieval errors are in good agreement above the $900 \mathrm{mb}$ level and are significantly smaller than the temperature variance, which means that the IASI temperature measurements are very informative. Good agreement between assessed and expected errors is an indicator that the averaging kernels adequately characterize the retrievals and that they can be used for retrieval assimilation using Rodgers' (2000) approach. Increase of the error below $900 \mathrm{mb}$ may be caused by undetected clouds or haze and an inaccurate modeling of the surface radiative properties.
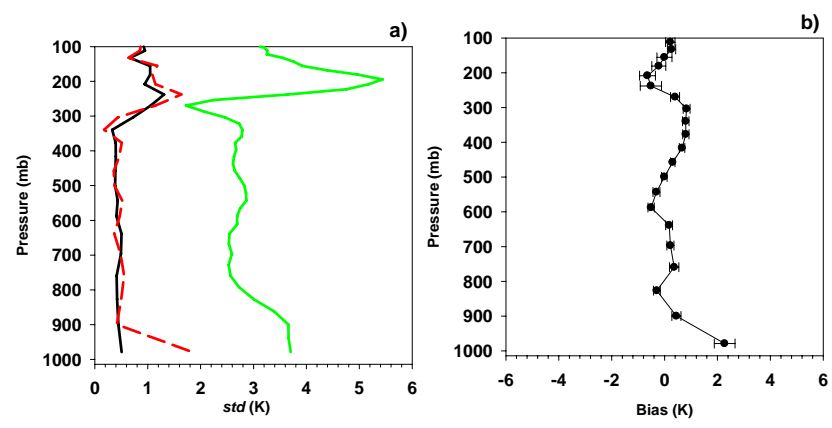

Fig. 4. Temperature retrieval errors. (a) black solid line is expected std error; red dashed line is the error assessed through validation; and green solid line is temperature profile variances as derived from radiosondes. (b) estimated bias, error bars indicate standard error.
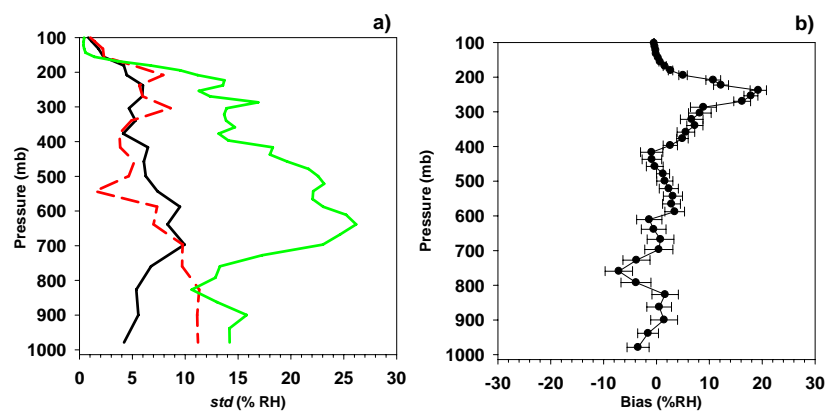

Fig. 5. Relative Humidity retrieval errors. (a) black solid line is expected std error; red dashed line is the error assessed through validation; and green solid line is temperature profile variances as derived from radiosondes. (b) estimated bias, error bars indicate standard error.

It is pertinent to note that the presented errors characterize the difference from the true atmospheric state on which the sounder is making its measurements. Estimated bias against radiosondes is within $\pm 0.5 \mathrm{~K}$ at most altitudes except in the tropopause and at the surface; error bars indicate standard error.

For relative humidity, the results are presented in Fig. 4. Similar to the temperature case, the assessed total retrieval error of relative humidity is in good agreement with the expected error at altitudes above $700 \mathrm{mb}$. The increase of the assessed error below $700 \mathrm{mb}$ is consistent with the same tendency in the temperature error, which may be an indicator that they have the same cause. Comparing the retrieval errors with the relative humidity profile variance shows that the sounding is informative in the troposphere above $800 \mathrm{mb}$. Estimated bias against radiosondes oscillates within $\pm 10 \%$ at most altitudes with significant error bars indicating standard error. 


\section{Conclusions}

The performed study demonstrates that for the most advanced atmospheric sounder - IASI, radiosonde data and the VAM can be efficiently used to accurately assess retrieval error in the presence of significant difference in characteristics of the compared systems and non-coincidence errors. Proper statistical characterization of the correlative data set allows accounting for the non-coincidence errors, and the averaging kernels can be used to reconcile the vertical resolution.

For temperature and water vapor retrievals, expected and assessed errors are in good agreement between $800-700 \mathrm{mb}$ and the tropopause. For temperature the std error of a single FOV retrieval is $\sim 0.6 \mathrm{~K}$ between $800-300 \mathrm{mb}$ with an increase to $\sim 1.5 \mathrm{~K}$ in tropopause and $\sim 2 \mathrm{~K}$ at the surface, possibly due to incorrect surface parameters and undetected clouds or haze. Bias against radiosondes oscillates within $\pm 0.5 \mathrm{~K}$ between $950-100 \mathrm{mb}$. For relative humidity, the $s t d$ of the error of a single FOV retrieval is below $10 \% \mathrm{RH}$ in the $800-300 \mathrm{mb}$ range; and bias is within $\pm 10 \% \mathrm{RH}$. The conclusion about the adequate characterization of the retrievals by the averaging kernels, in other words, agreement between the expected and actual error can be considered globally, whereas the numbers characterizing the errors are not representing the IASI global retrieval accuracy; they are pertinent to the conditions similar to the ones during the validation campaign.

Acknowledgements. IASI has been developed and built under the responsibility of the Centre National d'Etudes Spatiales (CNES, France). It is flown onboard the Metop satellites as part of the EUMETSAT Polar System. The IASI L1 data are received through the EUMETCast near real time data distribution service. This work was funded by the NPOESS IPO under NOAA contract DG133E-06-CQ-0079.

Edited by: T. Wagner

\section{References}

Barret, B., De Maziere, M., and Mahieu, M: Ground-based FTIR measurements of CO from Jungfraujoch: characterization and comparison with in situ surface and MOPITT data, Atmos. Chem. Phys., 3, 4857-4878, 2003,

http://www.atmos-chem-phys.net/3/4857/2003/.

Calbet, X., Schlüssel, P., Hultberg, T., Phillips, P., and August, T.: "Validation of the operational IASI level 2 processor using AIRS and ECMWF data", Adv. Space Res., 37, 2299-2305, 2006.

Eyre, J. R.: Variational assimilation of remotely-sensed observations of the atmosphere, J. Meteor. Soc., 75(1B), 331-338, 1997.

Global Space-based Inter-Calibration System (GSICS): http://www.orbit.nesdis.noaa.gov/smcd/spb/calibration/icvs/ GSICS/index.html, last access: 2008.
Larar, A. M., Smith, W. L., Zhou, D. K., Liu, X., Revercomb, H., Taylor, J. P., Newman, S. M., and Schlüssel, P.: IASI spectral radiance performance validation: case study assessment from the JAIVEx field campaign, Atmos. Chem. Phys. Discuss., 9, 10193-10234, 2009,

http://www.atmos-chem-phys-discuss.net/9/10193/2009/.

Lary, D. J. and Lait, L.: Using Probability Distribution Functions for Satellite Validation, Geoscience and Remote Sensing, IEEE T., 44(5), 1359-1366, 2006.

Liu, X., Zhou, D. K., Larar, A. M., Smith, W. L., Schlüssel, P., Newman, S. M., Taylor, J. P., and Wu, W.: Retrieval of C478 Atmospheric Profiles and Cloud Properties from IASI Spectra Using Super-Channels, Atmos. Chem. Phys. Discuss., 9, 86838736, 2009,

http://www.atmos-chem-phys-discuss.net/9/8683/2009/.

Migliorini, S., Piccolo, C., and Rodgers, C. D.: Intercomparison of direct and indirect measurements: Michelson Interferometer for Passive Atmospheric Sounding (MIPAS) versus sonde ozone profiles, J. Geophys. Res., 109, D19316, doi:10.1029/2004JD004988, 2004.

Pougatchev, N. S.: Validation of atmospheric sounders by correlative measurements, Appl. Opt., 47,,4739-4748, 2008.

Rodgers, C. D. and Connor, B. J.: Intercomparison of remote sounding instruments," J. Geophys. Res., 108(D3), 4116, doi:10.1029/2002JD002299, 2003.

Rodgers, C. D.: Retrieval of Atmospheric Temperature and Composition From Remote Measurements of Thermal Radiation, Rev. Geophys. Space Phys., 14, 609-624, 1976.

Rodgers, C. D.: Characterization and error analysis of profiles retrieved from remote sounding measurements, J. Geophys. Res., 95, 5587-5595, 1990.

Rodgers, C. D.: Inverse Methods for Atmospheric Sounding: Theory and Practice, World Scientific Publishing Co. Pte. Ltd., London, UK, 2000..

Schlüssel, P., Hultberg, T. H., Phillips, P. L., August, T., and Calbet, X.: The operational IASI Level 2 Processor, Adv. in Space Res., 36(12), 2299-2305, 2005.

Smith Sr., W. L., Zhou, D. K., Larar, A. M., Mango, S. A., Howell, H. B., Knuteson, R. O., Revercomb, H. E., and Smith Jr., W. L.: The NOESS Sounding Testbed Interferometer - Remotely Sensed surface and atmospheric conditions during CLAMS, J. Atmos. Sci., 62, 1118-1134, 2005.

Tobin, D. C., Revercomb, H. E., Knuteson, R. O., Lesht, B., Strow, L. L., Hannon, S. E., Feltz, W. F., Moy, L., Fetzer, E. J., and Cress, T: Atmospheric Radiation Measurement site atmospheric state best estimates for Atmospheric Infrared Sounder temperature and water vapor retrieval validation, J. Geophys. Res., 111, D09S14, doi:10.1029/2005JD006103, 2006.

Vaisala Radiosonde RS92-SGP brochure: http://www.vaisala.com/ files/Vaisala\%20Radiosonde\%20RS92-SGP\%20brochure\% 202005.pdf, last access: 2009.

Zhou, D. K., Smith, W. L., Larar, A. M., Liu, X., Taylor, J. P., Schlüssel, P., Strow, L. L., and Mango, S. A.: All weather IASI single field-of-view retrievals: case study - validation with JAIVEx data, Atmos. Chem. Phys., 9, 2241-2255, 2009, http://www.atmos-chem-phys.net/9/2241/2009/. 